\title{
Visible implant after augmentation rhinoplasty
}

\author{
II Gyu Kang, MD
}

Department of Otolaryngology-Head and Neck Surgery, Gachon University Gil Medical Center, Gachon University School of Medicine, Incheon, Rep. of Korea

\begin{abstract}
A 28-year-old female with thin nasal dorsal skin visited our department for augmentation rhinoplasty. We initially planned open rhinoplasty with a properly sized dorsal implant. However, the patient insisted on closed rhinoplasty. Thus, closed rhinoplasty was performed through a lower lateral-cartilage marginal incision, and augmentation was performed using a Gore-Tex implant. From the fourth day after the procedure, the white implant was seen through the skin of the nose, but was no longer visible at 1 month. This problem is common in young females with thin nasal skin. This case report reviewed the clinical features and literature on a visible implant after rhinoplasty.
\end{abstract}

Keywords: implant; rhinoplasty; thin skin; visible

\section{Introduction}

Augmentation implants used for rhinoplasty are usually made of silicone or expanded polytetrafluoroethylene (ePTFE). Displacement, malposition, extrusion, recurrent inflammation, and infection are frequently encountered after dorsal implantation of alloplastic material, as is short-nose deformity after silicone rhinoplasty, which is common and difficult to manage [1]. The most common undesirable outcome after ePTFE rhinoplasty is implant deviation (1.04\%), followed by a visible ePTFE implant $(0.47 \%)$, while implant infection only occurs in $0.38 \%$ of patients [2]. Implant visibility through nasal skin after insertion causes stress, especially in young female patients. However, little information is available on the frequency or duration of implant visibility through skin after rhinoplasty [2]. We describe a case in which implant visibility spontaneously resolved 1 month after rhinoplasty and provide a review of pertinent literature.

\section{Case report}

A 28-year-old female visited our department for augmentation rhinoplasty due to a small bony hump, an underprojected nasal tip, and an underprojected radix. She was noted to have thin skin. As the patient refused rhinoplasty through an external approach, we planned augmentation using a Gore-Tex implant (W. L. Gore \& Associates Inc., Flagstaff, AZ, USA) from the radix to the lower margin of the upper lateral cartridge after rasping the bony hump. We used a ready-made Gore-Tex implant. Tip augmentation was planned using crushed cartilage. Augmentation rhinoplasty was performed using an endonasal approach, as requested. An incision was made along the lower margin of the lateral crura of the lower lateral cartilage and extended to the medial crura of the lower lateral cartilage. Dissection was performed to the radix area in a supraperichondrial plane in the lateral cartilage of the nose, and in a subperiosteal plane in the nasal bone area. After dissection, a 5-mm-thick Gore-Tex implant was inserted through the marginal incision in the dis- 


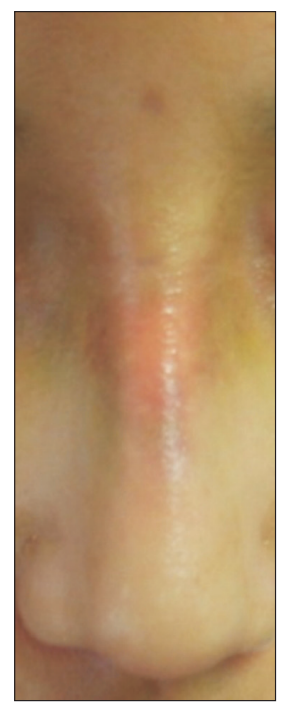

Fig. 1. Expanded polytetrafluoroethylene (Gore-Tex; W. L. Gore \& Associates Inc., Flagstaff, AZ, USA) implant visible through skin in rhinion area 4 days after rhinoplasty.

section plane. The operation was completed after suturing the incision site. The Gore-Tex implant was visible through skin at 4 days after surgery (Fig. 1) and remained visible for approximately 1 month, when it completely disappeared (Fig. 2).

\section{Discussion}

When silicone sheets are used for dorsal augmentation rhinoplasty, $3.7 \%$ of patients complain of implant visibility [3]. However, this does not refer to implant visibility through dorsal skin, but rather to a visible implant contour. Actually, few reports have investigated implant visibility through skin after nasal dorsal augmentation [2,3]. In our patient, the Gore-Tex implant on the dorsum was a little thick, presumably explaining the visibility through skin. The endonasal approach presents a greater risk that an implant will be contaminated with bacteria than the open approach; thus, we could not remove and adjust the thickness of the implant during surgery. Had an open approach been used, the implant could have been made thinner and replaced. In addition, a Gore-Tex implant, unlike silicone, tends to wrinkle when repeatedly inserted and removed from its pocket when the endonasal approach is used. Furthermore, a Gore-Tex implant is white, which makes it more visible through thin skin; if the skin has been stretched, the implant is easily

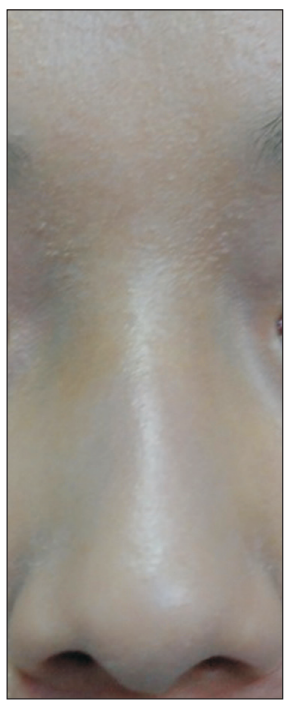

Fig. 2. Implant (Gore-Tex; W. L. Gore \& Associates Inc., Flagstaff, AZ, USA) no longer visible 1 month later.

seen and palpated. Because skin on the rhinion area is thinner than elsewhere on the nose, implants are often seen in this area. To prevent visibility after rhinoplasty, implants of appropriate thickness should be used and soft implants are preferred over hard block types. If it is likely to be visible through skin, the implant should be covered with fascia or soft tissue. In addition, dissection should be conducted in the supraperichondrial and subperiosteal planes.

\section{Conflicts of interest}

The author has nothing to disclose.

\section{References}

1. Jang YJ, Kim DY. Treatment strategy for revision rhinoplasty in Asians. Facial Plast Surg 2016;32:615-9.

2. Yap EC, Abubakar SS, Olveda MB. Expanded polytetrafluoroethylene as dorsal augmentation material in rhinoplasty on Southeast Asian noses: three-year experience. Arch Facial Plast Surg 2011;13:234-8.

3. Wang JH, Lee BJ, Jang YJ. Use of silicone sheets for dorsal augmentation in rhinoplasty for Asian noses. Acta Otolaryngol Suppl 2007;(558):115-20. 